\title{
Minitrack Introduction: Decision Analytics, Machine Learning, and Field Experimentation for Defense and Emergency Response
}

\author{
Alex Bordetsky \\ Naval Postgraduate School \\ abordets@nps.edu
}

\author{
Steven J. Mullins \\ Naval Postgraduate School \\ sjmullin@nps.edu
}

\author{
Bryan J. Hudgens \\ Naval Postgraduate School \\ bjhudgen@nps.edu
}

\section{Minitrack topic}

Defense and emergency first responders must make rapid, consequential decisions and machine learning can aid analytics to support these decisions. Machine learning offers enormous promise, yet wellpublicized struggles reveal the need for better datasets and for opportunities to learn in challenging settings. Field experimentation offers the potential to meet these needs through iterative interactions in complex scenarios. Field experimentation can provide live action to facilitate high fidelity datasets that can support machine learning and artificial/augmented intelligence applications. These experiments may incorporate participants from academia; government agencies; militaries; first responders at all levels; and global industry partners. This minitrack explores the interplay between machine learning, field experimentation, and optimization analytics, whether exploratory, theoretical, experimental, in such critical areas as Defense and Emergency Response.

\section{Salient research issues in this space}

- Requirements for design and implementation of machine learning in complex, collaborative network environments

- $\quad \mathrm{AI} / \mathrm{ML}$ models for networking, network operations, and networked decision support

- Decision Analytics and ML for organizational knowledge management
- $\quad \mathrm{AI} / \mathrm{ML}$ model transparency and evaluation in collaborative networks

- $\quad \mathrm{AI} / \mathrm{ML}$ for command and control

- Field experimentation for IoT applications

- Case studies illuminating any of the topics above

\section{Papers in this minitrack}

In the first paper, Little, Roberts, and Wallace explore how a pandemic's impacts on first responders and other critical personnel, as well as infrastructure and recovery supply chains, can affect a community's time to recover from a separate disaster. Their study suggests the impacts would be significant and would extend time to recover. The authors argue that such "compound extreme events" require additional attention to overcome our inadequate knowledge about how best to manage degraded capacity for critical personnel, infrastructures, and supply chains.

In the second paper, Shigley, Morton, and Tanner explore how efficiently organizations process incoming information, a topic of great importance to emergency response efforts. The authors assess the degree to which a power law distribution describes the amount of effort an organization invests in processing both important and inconsequential inputs. They hypothesize that increased focus on important inputs helps to defend organizational boundaries against external drivers. 